\title{
Hypothermic preservation of corneas in a hyperkalaemic solution (CPTES): II. Extended storage in the presence of chondroitin sulphate
}

\author{
MICHAEL J TAYLOR,' CHARLES J HUNT,' AND PETER W MADDEN 2 \\ From the 'MRC Medical Cryobiology Group, and 'University Departments of Surgery and Ophthalmology, \\ Cambridge CB2 $2 A H$
}

SUMMARY Periods of preservation for donor corneas, even for short times, are necessary to facilitate optimum conditions in penetrating keratoplasty. However, current techniques for corneal storage at low temperatures may not provide optimal conditions for maintaining tissue integrity. In particular, the ionic composition of the storage medium has received little attention since it has been assumed throughout that the normal complement of ions in tissue culture media will also be suitable for preservation at reduced temperatures. This study extends our previous investigations on the merits of using CPTES (corneal-potassium-TES), a potassium-rich balanced salt solution containing an impermeant anionic pH buffer (TES), as a storage solution specifically designed to prevent the loss of intracellular potassium and minimise endothelial cell swelling during the time that the normal regulatory processes are switched off. The effect of adding the natural polymer chondroitin sulphate (CS) as a colloid osmotic agent to the hyperkalaemic storage medium is now examined. Corneas stored in CPTES containing $2.5 \%$ chondroitin sulphate retained a very high level of structural and functional integrity after three, five, and seven days storage at $0^{\circ} \mathrm{C}$; furthermore, stromal swelling was restricted to only $21 \%$. All corneas stored in CPTES $+2.5 \%$ CS showed active endothelial function by thinning efficiently at rates that were greater than those previously reported for rabbit corneas stored for similar lengths of time in either M-K medium or K-sol. The zwitterionic buffers TES and HEPES were interchangeable in the hyperkalaemic solution and were non-toxic to corneal endothelium at a concentration of $100 \mathrm{mM}$. These compounds offer excellent $\mathrm{pH}$ buffering in bicarbonate-free medium.

Attempts to conserve the viability of donor biological tissues in vitro during the time interval that is inevitable between harvesting and transplantation rely on two fundamentally different approaches: one is to try to maintain normal physiological function during storage, while the other is to use physical or pharmacological means to depress function in a reversible way, that is to attain a state of 'suspended animation'. Although both are used at present to store corneas for penetrating keratoplasty, ' ' preservation by lowering temperature is most widespread, and techniques of cryopreservation offer the only practical means of achieving indefinite storage. ${ }^{13}$

Correspondence to Dr M J Taylor, MRC Medical Cryobiology Group, University Department of Surgery, Douglas House, Trumpington Road, Cambridge CB2 2AH.
As part of a broader study to improve methods of cryopreservation we have proposed the use of a hyperkalaemic balanced salt solution (CPTES)* to reduce both ionic imbalances and cell swelling during exposure at low temperatures when normal regulating mechanisms are retarded. ${ }^{+5}$ This solution has been shown to be an effective medium for introducing and removing multimolar concentrations of the cryoprotective agent dimethylsulphoxide. ${ }^{+h}$

This type of preservation solution can also provide benefits for intermediate-term storage at reduced temperatures since it is thought that it is unnecessary to support metabolism during hypothermia, and that

*CPTES: corneal-potassium-TES, a potassium-rich balanced salt solution containing the impermeant biological buffer compound $\mathrm{N}$ Tris (hydroxymethyl)methyl-2-amino ethane sulphonate. 
the normal distribution of intracellular water and ions can be controlled physically, rather than biochemically, by appropriate alterations to the composition of the bathing medium.' We have shown that CPTES was effective in maintaining the structural and functional integrity of isolated corneas stored for up to 5 days at $0^{\circ} \mathrm{C}$. $^{5}$ Furthermore, CPTES was significantly more efficient than an "extracellulartype' medium in restricting stromal swelling during the hypothermic storage period. Solutions used routinely in clinical practice for hypothermic storage of isolated corneas are all based on tissue culture medium having an 'extracellular' complement of ions, with the addition of colloid osmotic agents such as dextran, polyvinylpyrrolidone or glycosaminoglycans to help control corneal hydration.' The purpose of the present study was to evaluate the effects of adding chondroitin sulphate (CS) as an oncotic agent to the CPTES preservation medium. A comparative study which evaluates the role of polyvinylpyrrolidone (PVP) as the oncotic agent is described elsewhere."

As before, the effects of the hypothermic storage conditions on corneal integrity were assessed by measuring the change of thickness during storage, the ability of the endothelium to control stromal hydration during normothermic perfusion after storage, and by examining endothelial cell morphology and ultrastructure.

\section{Material and methods}

Details of the procedures used to isolate and manipulate corneas obtained from New Zealand White rabbits have been described previously. ${ }^{\text {th }}$ The method of storing isolated corneas in $5 \mathrm{ml}$ of preservation medium at $0^{\circ} \mathrm{C}$ has also been described.

\section{STORAGE CONDITIONS}

Groups of isolated corneas were stored at $0^{\circ} \mathrm{C}$ in CPTES supplemented with $2.5 \%(\mathrm{w} / \mathrm{v})$ chondroitin sulphate (Sigma grade III; $99 \%$ purity). Chondroitin sulphate was used without further purification. The formulation of CPTES buffer solution has been described in previous communications. ${ }^{+5}$ The combinations of storage solutions and storage times studied in this investigation are listed in Table 1. Although the effect of refrigerated storage in McCarey-Kaufman medium (M-K medium) has been studied extensively elsewhere, ${ }^{1}$ an additional group was included in this study for comparison with the new CPTES storage solution.

M-K medium was prepared as TC199 culture medium with Hanks's salts (Gibco) supplemented with $5 \%(\mathrm{w} / \mathrm{v})$ dextran-40. (Sigman Chemical Co. Product D-4133) as the oncotic agent." All storage
Table 1 Storage conditions studied

\begin{tabular}{lll}
\hline $\begin{array}{l}\text { Storage time } \\
\text { at } 0^{\circ} \mathrm{C} \text { (days) }\end{array}$ & $\begin{array}{l}\text { Base storage } \\
\text { medium }\end{array}$ & Oncotic agent \\
\hline 5 & TC199* & $5 \%$ Dextran-40* \\
3 & CPTES $\$$ & $2 \cdot 5 \%$ CS \\
5 & CPTES & $2 \cdot 5 \%$ CS \\
7 & CPTES & $2 \cdot 5 \%$ CS \\
$14^{\dagger}$ & CPTES & $2 \cdot 5 \%$ CS \\
7 & CPHEPES & $2 \cdot 5 \%$ CS \\
\hline
\end{tabular}

*M-K medium." †Examined for morphology only. $\ddagger$ CPTES $=$ Corneal-potassium-TES. ${ }^{*}$ \$Chondroitin sulphate (sodium salt Sigma C. 3254 . Grade III. $99 \%$ pure ): $2.5 \mathrm{~g}$ added to $10(0) \mathrm{ml}$ of medium. It was determined by dialysis and atomic adsorption spectrometry that an additional $4.5 \mathrm{mmols} \mathrm{Na}$ are contributed by incorporating $2.5 \% \mathrm{CS}$ in the storage media. CPHEPES=Cornealpotassium-HEPES, a potassium rich solution having the same composition as CPTES in which the TES is replaced with l(0) mmols/l of the buffer compound HEPES ( $\mathrm{N}$-2-hydroxy ethyl piperazine-N'-2-ethane sulphonic acid).

solutions were purged by filtration (Millipore, $0.22 \mu \mathrm{m}$ ) and where appropriate ( $>3$ days' storage) the media were supplemented with $0.1 \mathrm{mg} / \mathrm{ml}$ sodium carbenicillin (Pyopen, Beecham Research Laboratories) and $4 \mu \mathrm{g} / \mathrm{ml}$ amphotericin B (Sigma Chemical Co.) as antimicrobial agents. The series of experiments in which corneas were stored in solutions containing CS was carried out without the addition of antibiotics to the storage media. One cornea was held for 14 days in CPTES $+2.5 \%$ CS without any apparent contamination.

After storage the corneas were either mounted in the perfusion chamber of a specular microscope for monitoring changes in stromal hydration during perfusion at $34^{\circ} \mathrm{C}$, or were processed either for optical or for electron microscopy as previously described. ${ }^{\text {th }}$ During specular microscopy the corneas were normally perfused for a minimum of six hours with glutathione bicarbonate Ringer's solution (GBR); in some cases, however, as previously described, ${ }^{5}$ alternative perfusates were used for either the whole or part of the perfusion period in order to assess specific aspects of corneal function. For example, in some assessments the normal GBR perfusate was changed for a bicarbonate- and carbondioxide-free Ringer's solution (BCFR) in order to test the activity of the bicarbonate-dependent metabolic fluid pump in the endothelium. ${ }^{\text {th }}$ The composition of BCFR has been given previously.'

To demonstrate the activity of a bicarbonatedependent endothelial fluid pump, in those individual experiments when the cornea was perfused with GBR/ TES as previously described, ${ }^{5}$ it was necessary to use bicarbonate-free Ringer's solution which was also supplemented with $100 \mathrm{mmol} / \mathrm{l}$ TES. In this study, therefore, an additional solution (BCFR/TES) was 
formulated having the following composition (mmol/l): $\mathrm{KCl}(4 \cdot 83) ; \mathrm{NaCl}(30 \cdot 0) ; \mathrm{Na}_{2} \mathrm{HPO}_{4}$ (4.2); $\mathrm{NaH}_{2} \mathrm{PO}_{4} .2 \mathrm{H}_{2} \mathrm{O}(0 \cdot 66) ; \mathrm{Na}_{2} \mathrm{SO}_{4}(0 \cdot 6) ; \mathrm{MgCl}_{2}(0 \cdot 79)$; $\mathrm{CaCl}_{2}$ (1.03); $\mathrm{NaOH}(100.0) ; \mathrm{HCl}(30.0)$; glucose (4.99); reduced glutathione (1.0) and TES (100.0). The measured osmolality of this solution was 310 mosm. $\mathrm{kg}^{-1}$ and the $\mathrm{pH}$ was 7.4 after gassing with a mixture of $93 \% \mathrm{~N}_{2}$ and $7 \% \mathrm{O}_{2}$.

\section{MORPHOLOGY}

The gross structural integrity of the endothelial layer was examined by combined staining with trypan blue and alizarin red S (TB/AR). ${ }^{10}$ In general this vital staining assay was applied at the end of the storage period; however, in some cases as indicated below, the endothelium was stained after four or six hours' perfusion on the specular microscope. In this way a combined assessment of endothelial structure and function was achieved in the same cornea. Although specular microscopy itself allowed continual examination of endothelial morphology, this was restricted to a small central area, whereas dual staining with $\mathrm{TB} / \mathrm{AR}$ permitted structural evaluation of the entire endothelial layer.

Ultrastructural examination of endothelial cells in stored corneas was assessed either by transmission electron microscopy (TEM) as previously described,${ }^{46}$ or by scanning electron microscopy

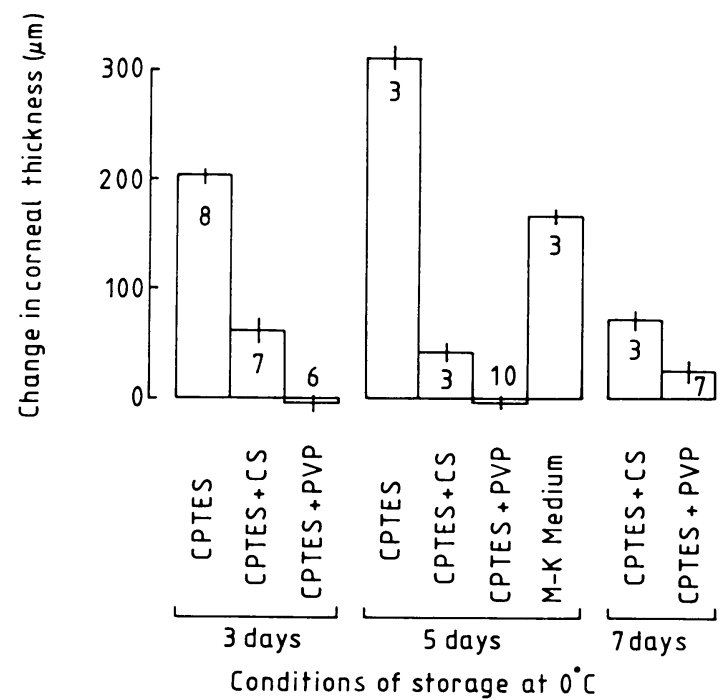

Fig. 1 The effect of polymeric additives in preventing stromal swelling during storage in CPTES at $0^{\circ} \mathrm{C}$. Each histogram represents the mean (bar $= \pm S E M$ ) change in corneal thickness for the number of corneas indicated in each group. For comparison, data for the changes in corneal thickness during storage in CPTES containing PVP-360 are included from a study published elsewhere. ${ }^{*}$
(SEM) after critical-point drying from ethanol, and sputter-coating."

\section{Results}

EXTENT OF CORNEAL SWELLING

DURING STORAGE

The marked effect of adding a colloid to CPTES solution to prevent stromal swelling during storage at $0^{\circ} \mathrm{C}$ is illustrated in Fig. 1. The addition of either PVP-360, described elsewhere, ${ }^{\times}$or chondroitin sulphate significantly reduced the extent of swelling during each of the storage intervals. The maximum increase in thickness with either of these colloids was only $27 \%$ after one week's storage. In contrast the mean thickness of corneas after five days' storage in either CPTES without added colloids, or in M-K medium, increased by $100 \%$ and $55 \%$ respectively.

\section{ENDOTHELIAL INTEGRITY AFTER STORAGE IN}

M-K MEDIUM

Corneas stored in $\mathrm{M}-\mathrm{K}$ medium for five days increased in thickness by 166 (SEM 4) $\mu \mathrm{m}(55 \%)$ and maintained this level throughout six hours' perfusion with GBR (Fig. 2). However, morphological examination showed that some damage to the endothelial layer had been sustained during storage. Fig. 3 shows that the integrity of the endothelial layer was compromised after five days' storage and that after seven days a great deal of intracellular disruption was evident on transmission electron microscopy.

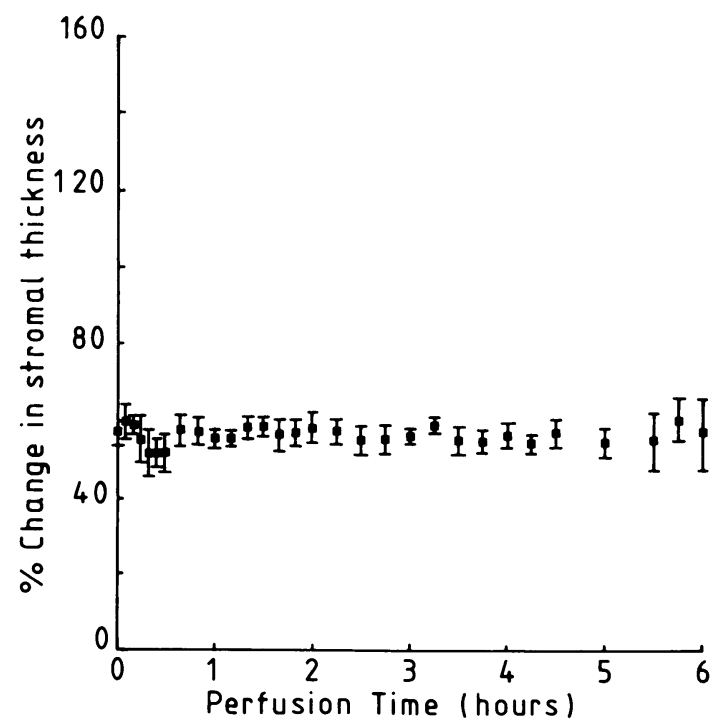

Fig. 2 Mean stromal thickness curves for a group of corneas following storage in $M-K$ medium for five days at $0^{\circ} \mathrm{C}(n=3)$. 


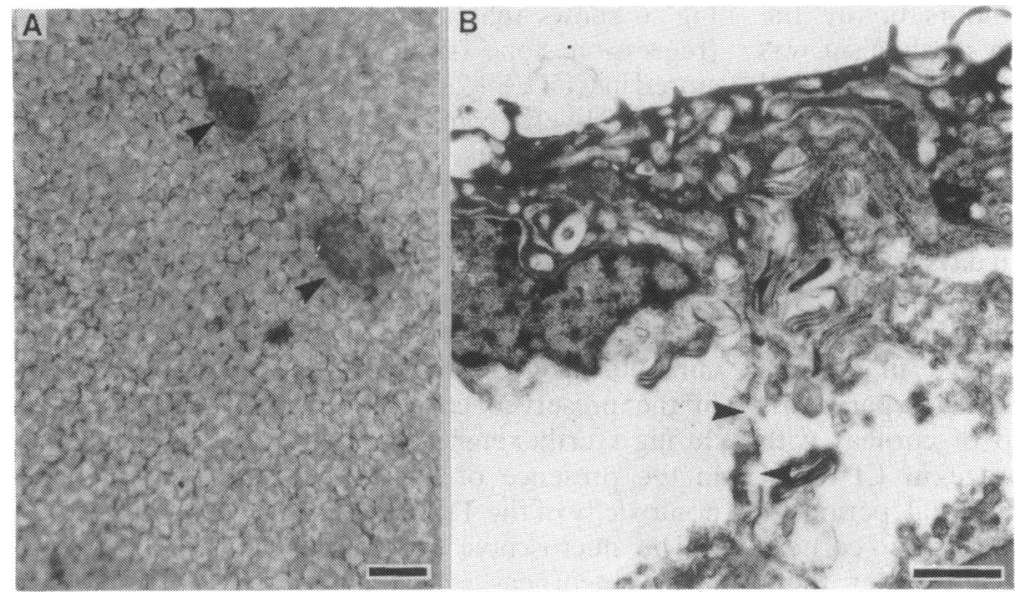

Fig. 3 Endothelial structure after storage in $M-K$ medium at $0^{\circ} \mathrm{C}$. A: Endothelial layer stained with $T B / A R$ after five days' storage showing that areas of the endothelium were disrupted, exposing the underlying basement membrane (arrowed). The remainder of the cell layer appears intact, but the individual cells had a 'rounded' profile instead of the normal angular and polygonal profile; this is probably due to endothelial cell swelling. Scale bar is $100 \mu \mathrm{m}$. B: TEM of endothelium after seven days' storage showing a disrupted endothelial layer with pronounced cytoplasmic swelling and the disruption of posterior and lateral plasma membranes (arrowed). Apical membranes and tight junctions remained intact. Scale bar is $I \mu \mathrm{m}$.

ENDOTHELIAL INTEGRITY AFTER STORAGE IN CPTES $+2 \cdot 5 \%$ CS

Groups of corneas stored for three, five, or seven days in CPTES $+2.5 \%$ chondroitin sulphate all showed a similar biphasic pattern of stromal

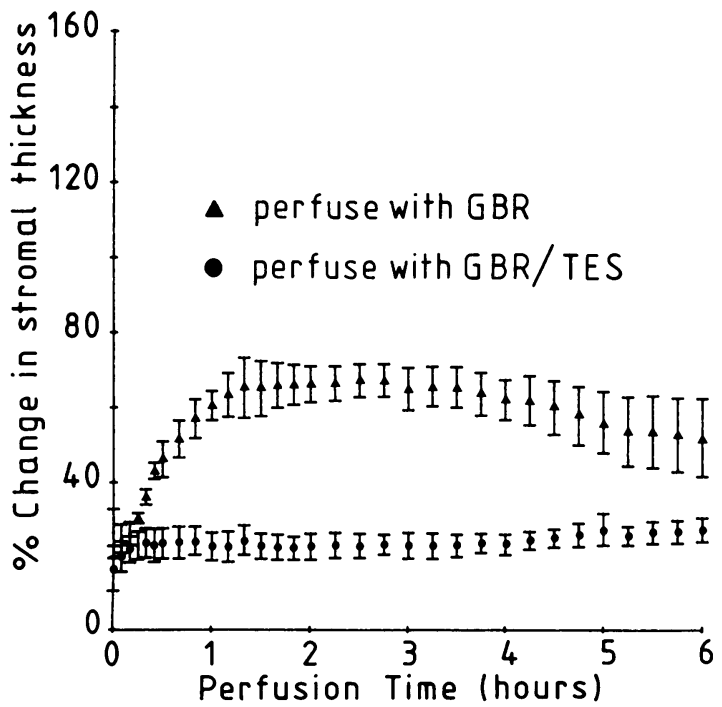

Fig. 4 Stromal thickness curves for groups of corneas stored in CPTES $+2.5 \%$ CS for three days at $0^{\circ} \mathrm{C}$. After storage the corneas were perfused under the specular microscope with either $G B R(\Delta)$ or $G B R / T E S(\bullet)$. The interpretation of these curves which represent the activity of fully functional endothelia is given in the text.
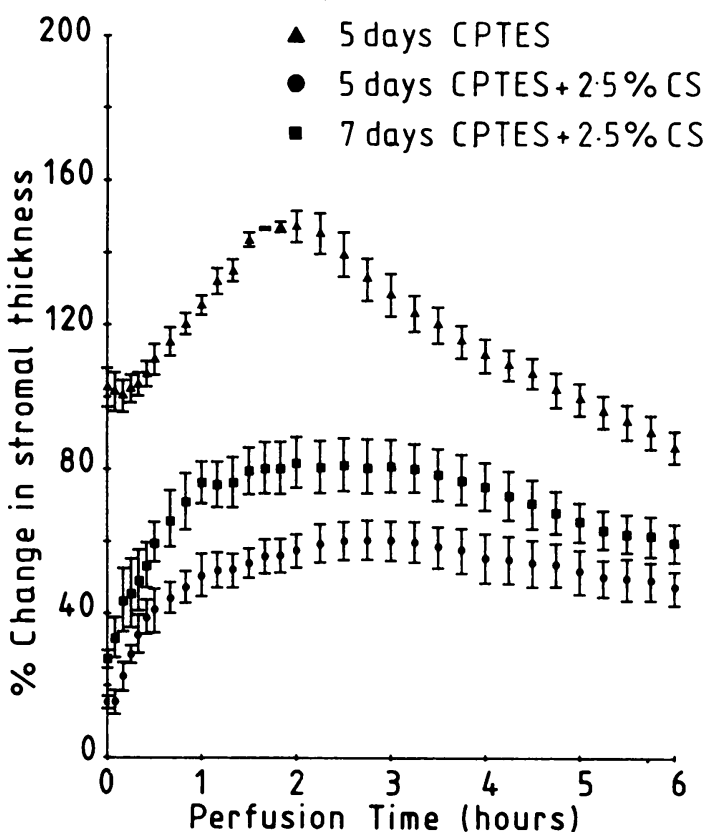

Fig. 5 Biphasic stromal thickness curves for groups of cornea preserved for $5(\bullet)$ and $7(\square)$ days in CPTES $+2.5 \%$ $C S(n=3)$. After the TES was eluted from the stroma during the first phase both groups of corneas thinned during the second phase of perfusion with GBR showing active endothelial function. The mean thickness curve for corneas stored for five days in CPTES without chondroitin sulphate $(\boldsymbol{\Delta})$ is shown for comparison $(n=3)$. The lower rate of thinning observed for corneas stored in medium containing CS is probably due to the persistent osmotic gradient established by the retention of exogenous $C S$ in the stroma as explained in the text. 
hydration changes during perfusion with GBR (Figs. 4 and 5). Each group of corneas increased in thickness for between one and two hours before the presence of an active dehydrating mechanism was evident. In each group the reversal of stromal swelling during the remainder of the perfusion clearly indicated the presence of a functional endothelium. The rates of stromal deturgescence during the latter part of the perfusion of groups of corneas that had been stored for three, five, or seven days in CPTES+ $2.5 \%$ CS are compared in Table 2 .

The osmotic response induced by the presence of TES in the tissue following storage in CPTES medium $^{s}$ was again confirmed in these experiments by perfusing an additional group of corneas with GBR/TES after three days' storage in CPTES+ $2.5 \%$ CS. Fig. 4 shows that the initial period of stromal swelling was abolished when stored corneas were perfused with GBR/TES, and under these circumstances the corneas maintained stromal thickness at a constant level through the perfusion (regression slope $=0 \cdot 1$ (standard error $0 \cdot 1) \% / \mathrm{h})$.

The functional viability of the endothelial layer in a cornea stored in CPTES + $2.5 \% \mathrm{CS}$ for three days and then perfused with GBR/TES was further demonstrated by reversible swelling of the stroma when the metabolic pump was inactivated during perfusion. Fig. 6 again shows that the presence of $100 \mathrm{mmol} / \mathrm{l}$ TES in the GBR perfusate prevents the initial swelling normally observed in corneas perfused with GBR without TES and that inhibition of the active bicarbonate-dependent pump, leading to stromal swelling, was achieved by substituting the GBR/TES perfusate with BCFR/TES. The reactivation of the endothelial pump and corneal thinning was also demonstrated by reverting to perfusion with GBR/TES.

Comparing this response, however, with that of the paired cornea which was stored in CPTES without chondroitin sulphate for the same length of time, we found the rates of change of stromal

Table 2 Stromal thinning in corneas preserved in CPTES $+2 \cdot 5 \%$ chondroitin sulphate at $0^{\circ} \mathrm{C}$.

\begin{tabular}{llllll}
\hline Storage time (days) & $\begin{array}{l}\text { Mean initial } \\
\text { thickness }{ }^{*} \text { in } \mu \mathrm{m} / \mathrm{h} \\
\text { (SEM) }\end{array}$ & \multicolumn{2}{l}{ Rate of thinning } \\
\cline { 3 - 5 } & \multicolumn{2}{l}{$\% / h(S E M) \mu m$} \\
\hline 3 & 283 & $(12)$ & $5 \cdot 2$ & $(0 \cdot 3)$ & $14 \cdot 7$ \\
5 & 288 & $(7)$ & $4 \cdot 4$ & $(0 \cdot 1)$ & $12 \cdot 7$ \\
7 & 261 & $(7)$ & $7 \cdot 8$ & $(0 \cdot 3)$ & $20 \cdot 3$ \\
\hline
\end{tabular}

*Thickness of cornea measured within 60 min of enucleation: this is the value to which all subsequent changes in thickness are normalised. thickness during the periods of perfusion with both GBR/TES and BCFR/TES to be noticeably different. Fig. 6 shows that the swelling and thinning rates (regression slope (standard error)) for the cornea stored in CPTES $+2.5 \%$ CS were $33(1)$ and $6(2) \mu \mathrm{m} /$ $\mathrm{h}$ respectively compared with the swelling and deturgescence rate of 25 (1) $\mu \mathrm{m} / \mathrm{h}$ for the cornea stored in CPTES alone. The implication of these data, that storage in medium containing chondroitin sulphate in some way influences the subsequent rate of change of hydration under the influence of a functioning endothelium is discussed below. The curves in Fig. 6 also demonstrate that the endothelium in the preserved corneas remains fully functional during a further eight hours' normothermic perfusion in the presence of $100 \mathrm{mM}$ TES, confirming the nontoxicity of the TES buffer.

The microscopic appearance of the endothelial layer in corneas stored in CPTES $+2.5 \% \mathrm{CS}$ for three, five, and seven days is shown in Figs.7-9 respectively. It is clearly apparent that in all cases the

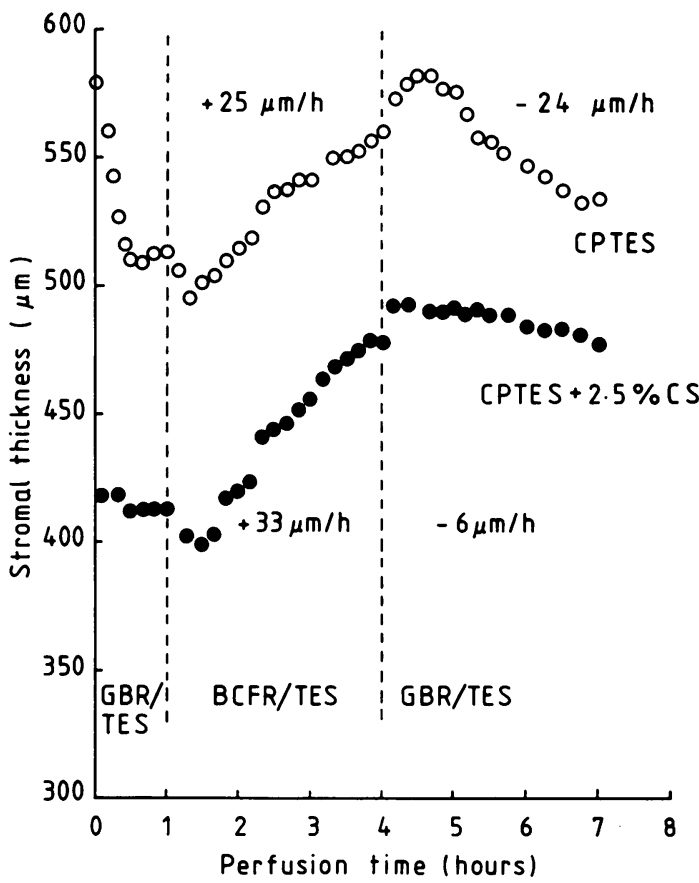

Fig. 6 Stromal thickness curves demonstrating fully functional endothelia in a pair of corneas preserved for three days at $0^{\circ} \mathrm{C}$ in either CPTES (०) or CPTES $+2.5 \%$ CS (•). Stromal swelling and thinning rates are indicated for the periods during which the endothelial pump is switched off and then reactivated during normothermic perfusion with either BCFR/TES or GBR/TES respectively. See text for further discussion of these curves. 


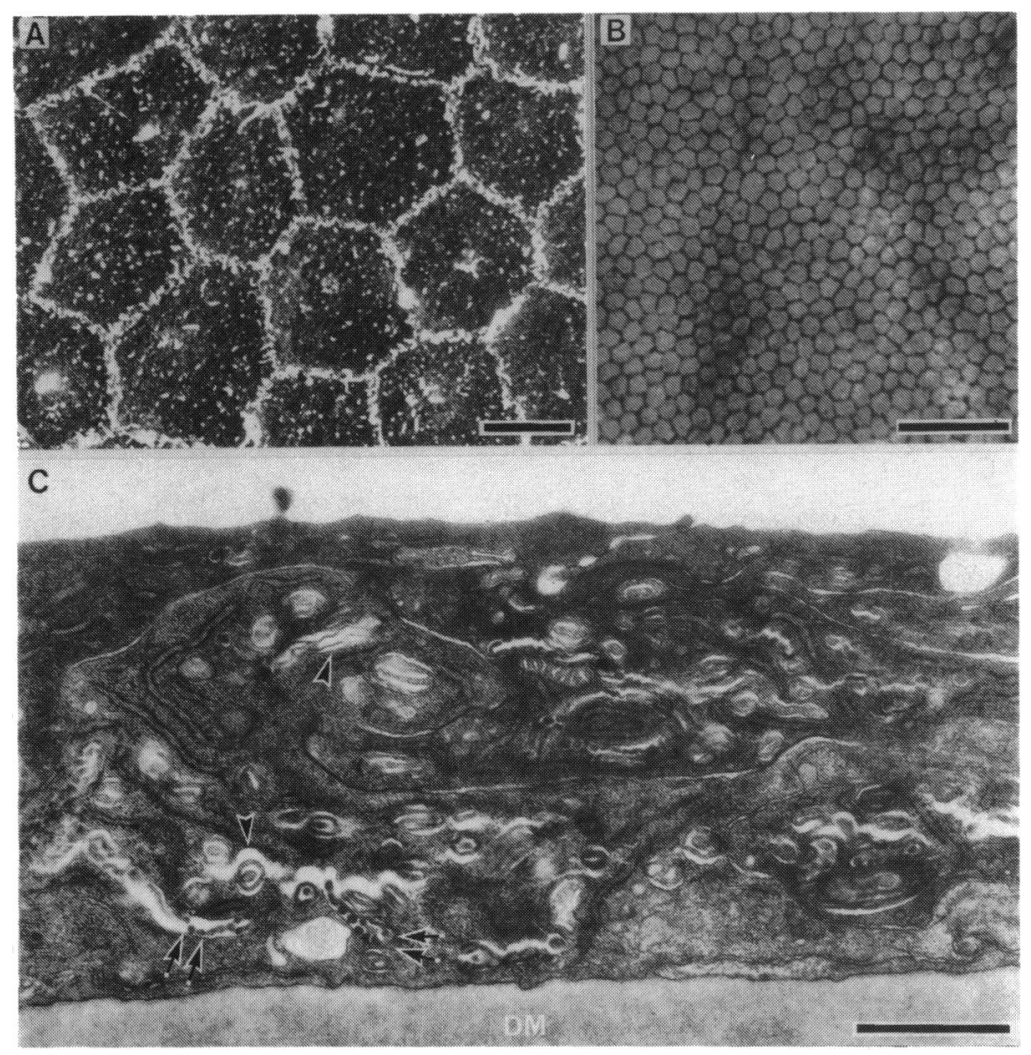

Fig. 7 Corneal endothelium after three days' storage at $0^{\circ} \mathrm{C}$ in CPTES $+2 \cdot 5 \%$ chondroitin sulphate. A: SEM of intact endothelial layer immediately following the storage period. The cells show only slight lifting of the apical flaps and a nearly normal complement of microvillae. $\mathrm{B}$ : TB/AR staining showing a nearly normal endothelial cell layer with no loss of cells and no uptake of trypan blue. C: TEM shows an intact, normal endothelial layer closely apposed to Descemet's membrane (DM). only slight swelling of the mitochondrial outer compartment (arrowheads) and the presence of beaded forms with swollen cristae (double arrows) is apparent. Scale bar: A: $10 \mu \mathrm{m}$; B: $100 \mu \mathrm{m}$; C: I $\mu \mathrm{m}$.

endothelial mosaic is well preserved, and Fig. 10 shows that even after 14 days' storage very little damage was detectable. Scanning electron microscopy confirmed that an intact cell layer was present at the end of each storage period and that damaged cells were confined to areas of the cell layer in the vicinity of ridges or folds. Transmission electron microcopy showed near normal endothelial ultrastructure after three days' storage, with only slight mitochondrial swelling apparent. As storage time increased, further changes occurred to the endothelium: though a few cells showed increased Golgi activity and a proliferation of the apical cytoplasm and there was some slight intracellular oedema, most cells had only modest and reversible structural changes even after seven days' storage.

Replacement of TES in the hyperkalaemic solution with an alternative zwitterionic buffer $(100 \mathrm{mmol} / \mathrm{l}$ HEPES; Table 1) also resulted in excellent preservation of corneal structure and function after seven days' storage at $0^{\circ} \mathrm{C}$. Fig. 11 shows the presence of an active fluid pump in the endothelium of a cornea stored for seven days in CPHEPES $+2 \cdot 5 \%$ CS. This cornea also had a morphologically intact endothelial layer at the end of the storage and perfusion period (Fig. 10).

\section{Discussion}

The rationale for using a potassium-rich solution containing an impermeant anion with good hydrogen ion buffering for hypothermic preservation of corneas has been described at the beginning of this report and in previous publications. ${ }^{+5}$ Moreover, it has been shown that isolated corneas are better preserved at $0^{\circ} \mathrm{C}$ in the 'intracellular-type' solution, CPTES, than in the 'extracellular-type' solution GBR. ${ }^{5}$ Further studies have now been undertaken to assess the value of adding colloid osmotic agents to the hyperkalaemic storage medium.

In the present study the survival of corneal endothelium after storage in CPTES solution containing the natural polymer chondroitin sulphate is assessed for comparison with a parallel study that evaluated the merits of polyvinylpyrrolidone (PVP) as an oncotic agent. ${ }^{\times}$Chondroitin sulphate has been used in recent years as an ingredient of other corneal storage solutions. ${ }^{12-14}$ 
Fig. 8 Corneal endothelium after five days' storage at $0^{\circ} \mathrm{C}$ in CPTES $+2 \cdot 5 \%$ CS. A: SEM shows an intact cell layer with evidence of active cellular ingrowth (rosette formation-arrow). Many cells had a 'cobblestone'-like appearance, with a central bulge (arrowheads). Apical flaps were swollen. B: Good preservation of the endothelial mosaic as delineated by staining with alizarin red $S$. No cells stained with trypan blue. C and D: TEM immediately following storage. The endothelium remains nearly normal. A pical flaps are raised but tight junctions remain closed. There is more pronounced mitochondrial swelling in places (C, arrowheads) and the appearance of some condensed forms (D, arrowhead); smooth endoplasmic reticulum is swollen (D arrow). In some areas there is a supranuclear proliferation of vesicles which may correlate with the bulges seen in the SEM. Scale bar A: $10 \mu \mathrm{m}$; B: $100 \mu \mathrm{m}$; C. D: $1 \mu \mathrm{m}$.
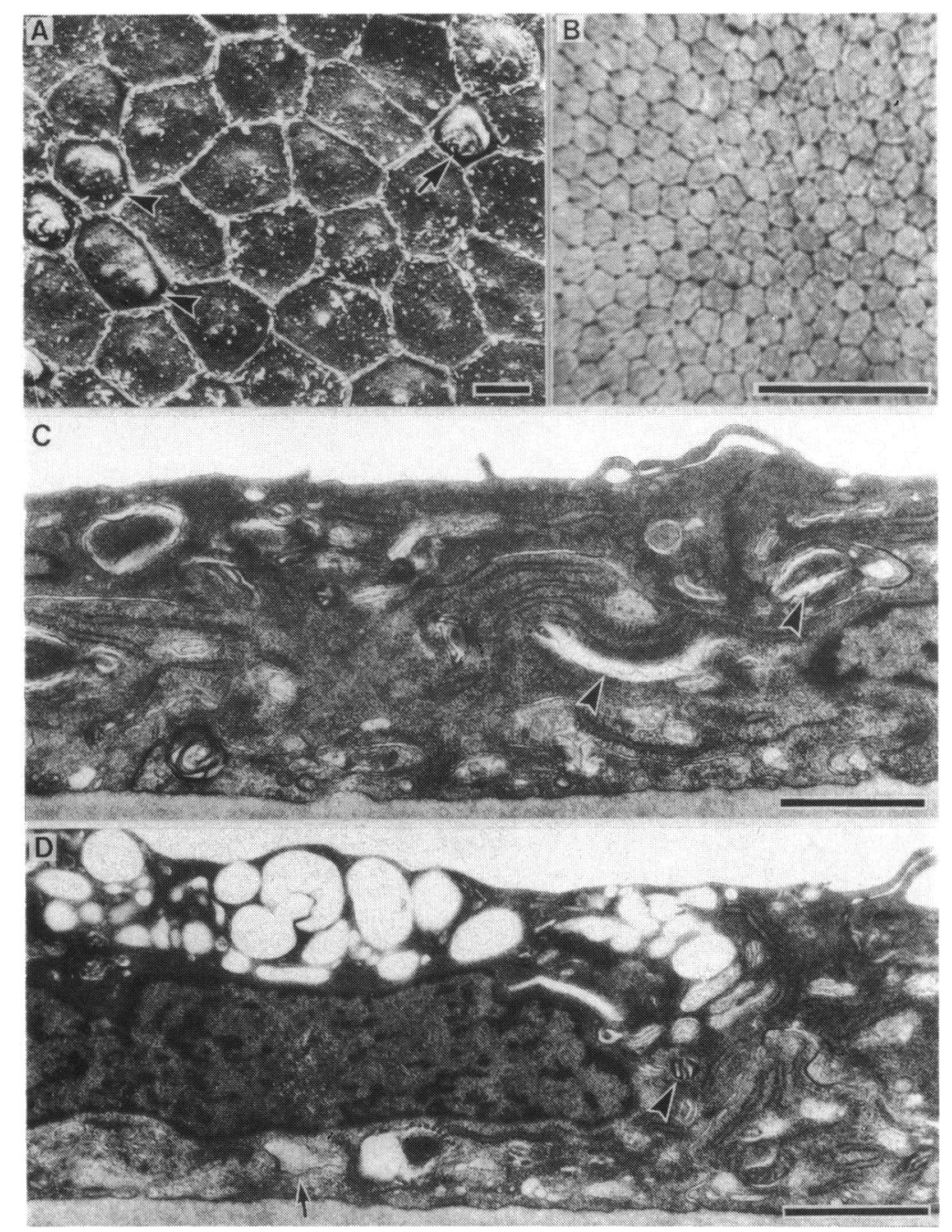

ADDITION OF ONCOTIC AGENTS TO CORNEAL STORAGE MEDIA

A variety of oncotic agents have been used to supplement corneal storage solutions and effectively restrict stromal swelling during hypothermic preservation.' Polyvinylpyrrolidone (PVP) has had only limited use as a colloid in hypothermic storage solutions for corneal preservation at refrigerator temperatures, ${ }^{15}$ and we have recently reported elsewhere ${ }^{x}$ that PVP-360 (average molecular weight 360000 daltons) is extremely effective in preventing any stromal swelling in corneas stored at $0^{\circ} \mathrm{C}$ for up to five days and restricts swelling to less than $10 \%$ of the original thickness in those corneas stored for up to seven days. However, even though the gross struc- tural integrity of the endothelial layer was apparently well preserved, corneas stored for longer than three days in CPTES containing 7\% PVP-360 did not retain good endothelial function, and the endothelial ultrastructure revealed a number of detrimental changes; these were probably related to the pinocytotic uptake of the polymer.*

Dextran has also been used as a colloid osmotic agent in corneal preservation solutions, ${ }^{4 / \mathrm{in}}$ but as shown in this study it is clearly not as effective in restricting stromal swelling as PVP-360 or chondroitin sulphate. Endothelial function was better preserved after storage for five days in $\mathrm{M}-\mathrm{K}$ medium containing dextran-40 than in CPTES containing PVP-360, ${ }^{\circ}$ but a number of detrimental structural changes were 

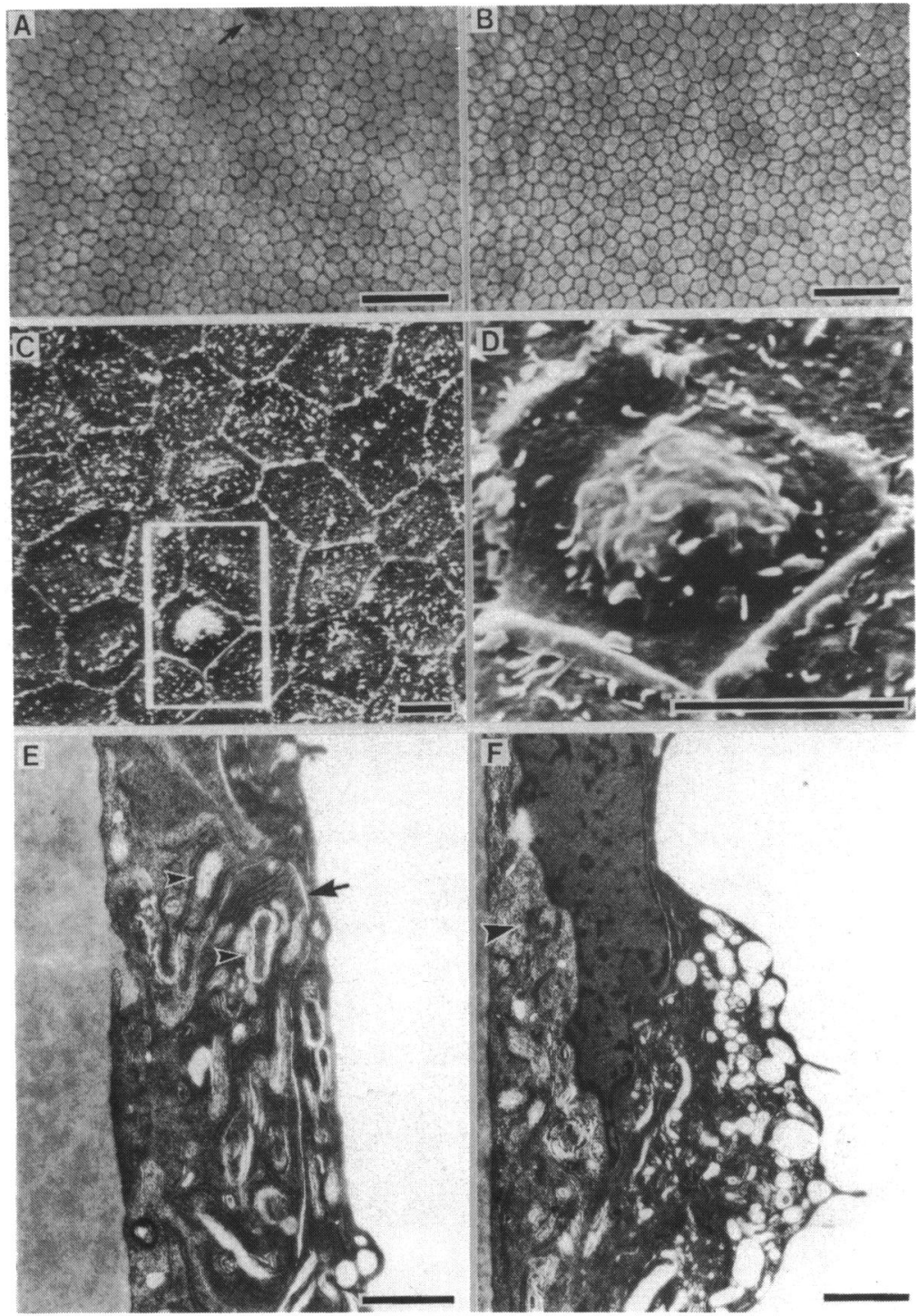

Fig. 9 Corneal endothelium after seven days' storage in CPTES+ $2.5 \%$ chondroitin sulphate. A: Excellent endothelial morphology revealed by $T B / A R$ staining immediately after seven days' preservation. Only a single cell had become permeable to trypan blue (arrow). B: Complete endothelial layer integrity was retained after rewarming to $34^{\circ} \mathrm{C}$ and perfusion under the specular microscope for $4 \mathrm{~h}$ during the functional assessment shown in Fig. 5. C: SEM of endothelial layer; note increased number of microvillae. D: Higher magnification of area outlined in C showing cellular protrusion with numerous blebs and microvillae. E: TEM showing continued maintenance of nearly normal morphology with slight widening of intercellular space (arrow) and altered mitochondria (arrowheads). F: Area of vesicular proliferation related to increased Golgi activity, which may correlate with the cellular protrusions seen by SEM; note slight basel oedema (arrowhead). Scale bar: A, B: 100 $\mu \mathrm{m} ; \mathrm{C}, \mathrm{D}: 10 \mu \mathrm{m} ; \mathrm{E}, \mathrm{F}: 1 \mu \mathrm{m}$. nevertheless observed after five and seven days' storage. Recent reports by other workers using human corneas also show structural damage in corneas rewarmed to $37^{\circ} \mathrm{C}$ after storage in $\mathrm{M}-\mathrm{K}$ medium for up to six days. ${ }^{17}$

SUPERIORITY OF CHONDROITIN SULPHATE

The most striking observation in this study was the superior quality of corneas stored in CPTES containing $2.5 \%(\mathrm{w} / \mathrm{v})$ chondroitin sulphate after any of the storage periods. Chondroitin sulphate, which is a negatively charged glycosaminoglycan with an average molecular weight of approximately 40000 daltons (range 25000-80000), was very effective in helping to maintain stromal hydration during hypothermic storage and, as with storage in CPTES+ PVP-360, ${ }^{\circ}$ corneas were thin and perfectly transparent at the end of storage. However, in contrast to corneas stored in CPTES + PVP-360 those stored at $0^{\circ} \mathrm{C}$ in CPTES with $2.5 \% \mathrm{CS}$ also retained a fully functional endothelium, as indicated by stromal thinning during subsequent perfusion on the specular microscope.

The functional ability of corneas stored in CPTES $+2 \cdot 5 \%$ CS was further demonstrated by the reversible swelling induced by inhibiting and then 
Fig. 10 Structural integrity of corneal endothelium after seven and 14 days' storage in hyperkalaemic preservation solutions containing chondroitin sulphate. A: Seven days' storage in CPTES $+2.5 \%$ CS. The entire endothelial layer was intact and all cells were viable by trypan blue exclusion. A few giant multinucleate cells were observed as shown here. B: Seven days' storage in CPHEPES $+2 \cdot 5 \%$ CS showing a normal mosaic of endothelial cells covering the entire corneal button. C: Fourteen days. storage in CPTES+2.5\% CS. The cell layer was intact and sites of individual cell replacement were apparent (arrows). Occasional enlarged cells were also observed. D: Fourteen days' preservation in CPTES $+2 \cdot 5 \%$ CS showing that only minor injury was sustained during storage under these conditions; a few single cells are lost from the endothelial layer and replaced by neighbouring healthy cells (arrow). Scale bar is $100 \mu \mathrm{m}$.
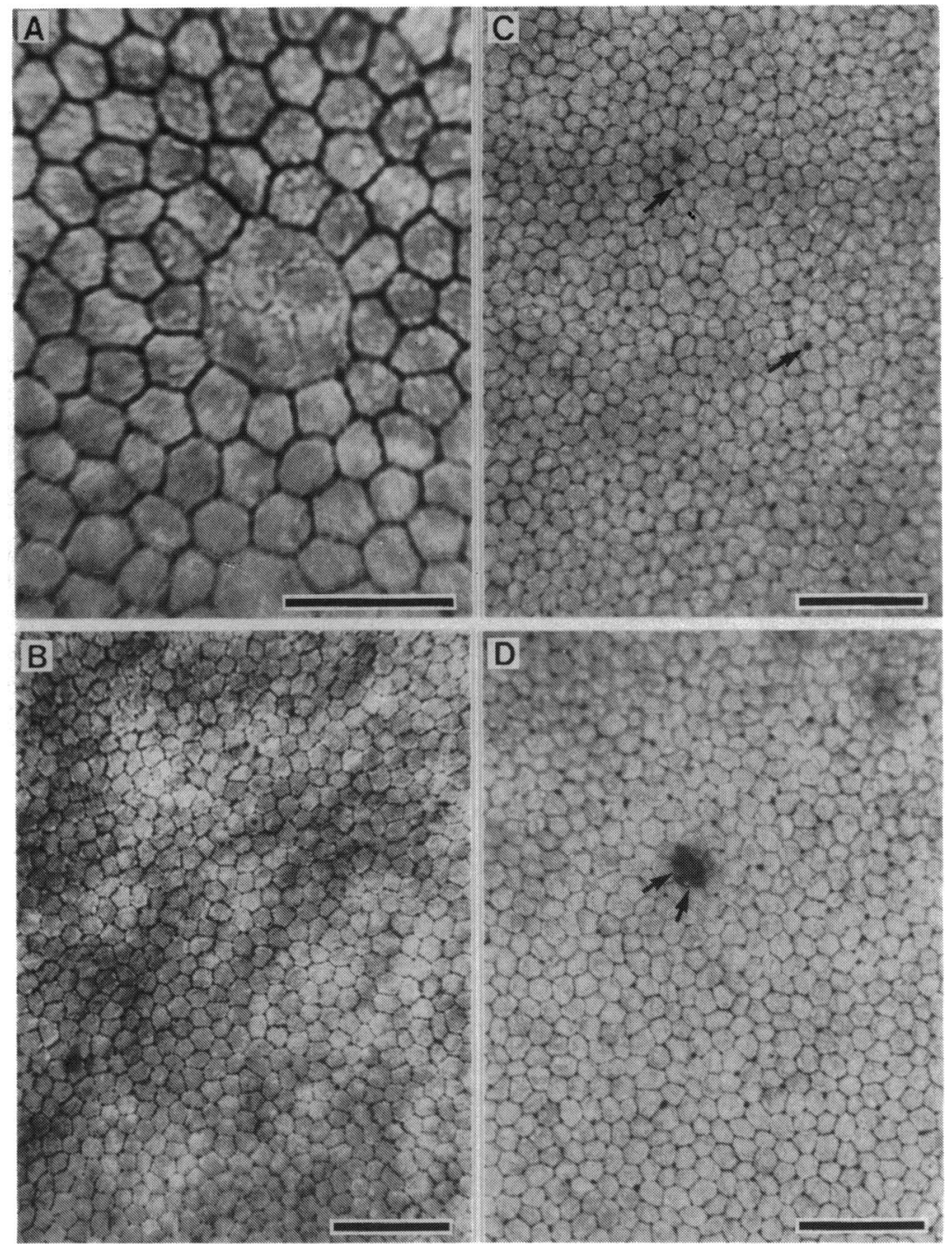

reactivating the bicarbonate-dependent pump of the endothelium. It was noted, however, that the rates of swelling when the pump was switched off was greater, and the rate of thinning after reactivation was less, than the values recorded for the paired cornea stored for the same length of time in CPTES without chondroitin sulphate. Furthermore, the mean rate of thinning during perfusion for groups of corneas stored in CPTES + $2 \cdot 5 \%$ CS for five days was less than for a comparable group stored in CPTES without chondroitin sulphate (see Fig. 5). ${ }^{5}$ These observations are consistent with those previously reported by Hull et al, who noted that rabbit corneas stored at $+4^{\circ} \mathrm{C}$ in $\mathrm{K}$-sol containing $2.5 \%$ CS failed to 'temperature reverse'; in contrast corneas stored in
M-K medium thinned at 6 or $9 \mu \mathrm{m}$-h after storage for three and seven days respectively. ${ }^{12}$ They postulated that the shorter chain length moieties of chondroitin sulphate penetrate the stroma more rapidly than the longer chain length molecules, giving rise to an increased osmotic force across the endothelium. In our experiments this would account for the higher rate of swelling in corneas whose bicarbonate pump was switched off during perfusion following storage in CPTES $+2.5 \%$ CS. Moreover, it is proposed that, because chondroitin sulphate is a natural glycosaminoglycan, normally present in corneal stroma, exogenous chondroitin sulphate may be sequestered in the stroma and eluted more slowly than foreign polymers such as dextran following 


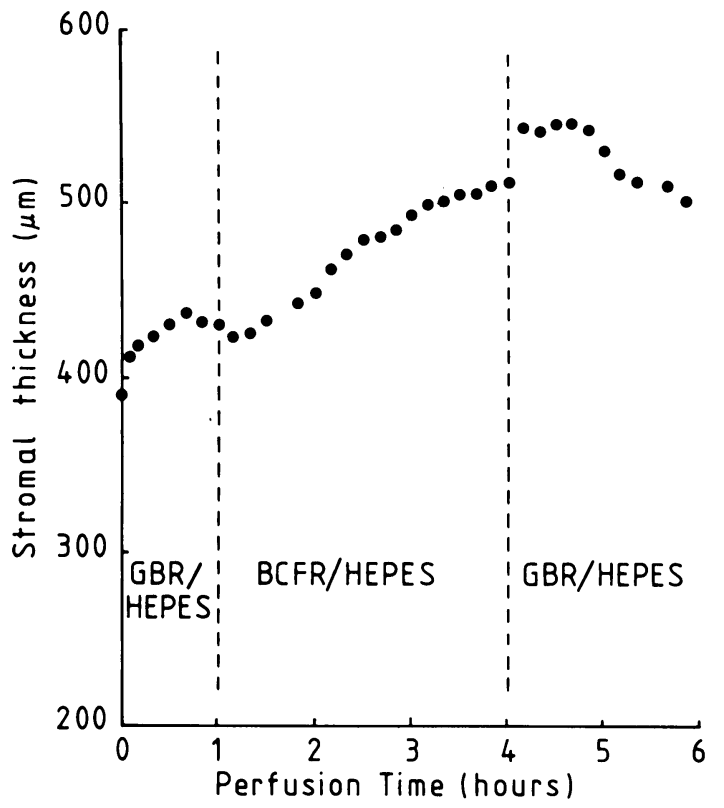

Fig. 11 Functional viability of a cornea preserved for seven days in CPHEPES $+2.5 \%$ chondroitin sulphate at $0^{\circ} \mathrm{C}$. Reversible swelling of the stroma is demonstrated by alternate perfusion in GBR/HEPES and BCFR/HEPES, which enables the endothelial bicarbonate-dependent pump to be switched off and reactivated as described in the text. This physiological test was conducted at $34^{\circ} \mathrm{C}$ in the presence of $100 \mathrm{mmol} / \mathrm{HEPES}$, showing the non-toxicity of this buffer compound.

storage. A persistent osmotic gradient would therefore be established across the endothelium of corneas stored in CPTES + CS resulting in the lower rates of thinning observed during subsequent perfusion with GBR or GBR/TES compared with corneas stored in CPTES alone.

On this basis the profile of the stromal swelling curves for groups of corneas stored for three, five, or seven days in CPTES $+2.5 \%$ CS (Figs. 4 and 5 ) might be explained in the following way. Initially corneas are thin, having swollen by less than $20 \%$ during storage. The stroma then swells during the first one to two hours of perfusion owing to the osmotic gradient established by the presence of both TES and exogenous chondroitin sulphate which have permeated the stroma during storage. TES will be eluted more rapidly (within $80-90 \mathrm{~min}$ as shown previously ) than chondroitin sulphate, which is probably bound in some way, resulting in a persistent osmotic gradient which increases the rate of passive permeation of water counteracting the active dehydrating mechanism of the functioning endothelium. After elution of the TES all corneas stored in CPTES $+2 \cdot 5 \% \mathrm{CS}$ showed an active thinning of the stroma during the latter part of the perfusion, and the rates of thinning were greater than those previously reported for rabbit corneas stored for similar lengths of time in either M-K medium of K-sol. ${ }^{12}$

The use of glycosaminoglycans in corneal storage solutions was proposed over 20 years ago by groups of Japanese workers who reported successful preservation for 1 week.' More recently a number of experimental and clinical reports have advocated or supported the inclusion of chondroitin sulphate in both low temperature preservation solutions such as $\mathrm{K}$-sol ${ }^{12-1+1018}$ and organ culture media for storage at $37^{\circ} \mathrm{C}^{19}{ }^{12}$ The present study confirms the superiority of chondroitin sulphate as a non-toxic colloid osmotic agent for restricting stromal swelling and for maintaining endothelial cell structure and function during refrigerated storage. Moreover, these experiments support our previous proposal that during hypothermic storage it is unnecessary to support metabolism by bathing corneas in solutions based on tissue culture media and that optimum maintenance of the ionic and hydraulic balance within corneal endothelium is more likely to be achieved at reduced temperatures by using an 'intracellular' type solution such as CPTES. ${ }^{45}$

Additional benefits such as the superior $\mathrm{pH}$ buffering capacity of CPTES and CPHEPES remains to be evaluated in further studies. ${ }^{212}$ However, it is well known that the structure and physiological function of corneal endothelium is sensitive to the ambient $\mathrm{pH}^{2.3-27}$ The control of $\mathrm{pH}$ during tissue storage is therefore, extremely important for optimum preservation, and, although this particular aspect of corneal preservation has received little attention, it has been reported that $\mathrm{pH}$ is better controlled in some corneal storage solutions than in others..$^{2 \times 2 y}$ The presence of $100 \mathrm{mmol} / \mathrm{l}$ of a zwitterionic buffer such as TES or HEPES in this hyperkalaemic preservation medium means that their hydrogen-ion buffering capacity in the physiological range will be better than any other corneal storage or irrigating solution, and, as discussed in our previous communication, ${ }^{5}$ the role of changes in $\mathrm{pH}$, especially in bicarbonate-free medium, demands further study.

Technical support for these experiments was provided by Mr David Chapman, whose care and skills we acknowledge with gratitude.

\section{References}

1 Taylor MJ. Clinical cryobiology of tissues: preservation of corneas. Cryobiology 1986: 23: 323-53.

2 Bourne WM, Doughman DJ, Lindstrom RL. Decreased endothelial cell survival after transplantation of corneas preserved by three modifications of corneal organ culture technique. Ophthalmology 1985; 92: 1538-41. 
3 Taylor MJ. Sub-zero preservation and the prospect of long-term storage of multicellular tissues and organs. In: Calne RY, ed. Transplantation immunology-clinical and experimental. Oxford: Oxford University Press, 1984: chapter 22: 36(1-90).

4 Taylor MJ, Hunt CJ. A new preservation solution for storage of corneas at low temperatures. Curr Eye Res 1985; 4: 963-73.

5 Taylor MJ. Hunt CJ. Hypothermic preservation of corneas in a hyperkalaemic solution (CPTES): 1. Short-term storage in the absence of colloid osmotic agents. Br J Ophthalmol 1989; 73: 781-91.

6 Taylor MJ, Hunt CJ. Tolerance of corneas to multimolar dimethylsulfoxide at $0^{\circ} \mathrm{C}$ : Implications for cryopreservation. Invest Ophthalmol Vis Sci 1989; 30: 40)(12.

7 Pegg DE. Principles of tissue preservation. In: Morris PJ, Tilney NL, eds. Progress in transplantation. Edinburgh: Churchill Livingstone, 1985: chapter 4: 69-105.

8 Taylor MJ, Hunt CJ. The effect of polyvinylpyrrolidone as a colloid osmotic agent in a hypothermic preservation solution for corneas. Cryo-Letters 1989; 10: 235-48.

9 McCarey BE, Kaufman HE. Improved corneal storage. Invest Ophthalmol Vis Sci 1974; 13: 165-73.

10) Taylor MJ. Hunt CJ. Dual staining of corneal endothelium with trypan blue and alizarin red $\mathrm{S}$ : importance of $\mathrm{pH}$ for the dyelake reaction. BrJ Ophthalmol 1981; 65: 815-9.

11 Madden PW. The evaluation of endothelial damage following corneal storage: a comparison of staining methods and the value of scanning electron microscopy. Curr Eye Res 1987; 6: 1441-51.

12 Hull DS, Green K, Frey N. Rabbit corneal endothelial physiologic and morphologic characteristics following storage in MK medium and K-SOL. Acta Ophthalmol Kbh 1986; 64: 649-56.

13 Bourne WM. Endothelial cell survival on transplanted human corneas preserved at $4{ }^{\circ} \mathrm{C}$ in $2.5 \%$ chondroitin sulfate for 1 to 13 days. Am J Ophthalmol 1986: 102: 382-6.

$14 \mathrm{Yau}$ CW, Kaufman HE. A medium-term corneal preserving medium. (K-SOL) Arch Ophthalmol 1986; 104: 598-601.

15 Schimmelpfenning B. Corneal Preservation at $-4^{\circ} \mathrm{C}$ in a modified tissue culture medium. Graefes Arch Clin Exp Ophthalmol 1978; 206: 57-66.

16 Tamaki K, Yamaguchi T, Varnell ED, Kaufman HE. Histological study of corneas preserved in two new media. $\mathrm{Br} \mathrm{J}$ Ophthalmol 1987: 71: 570-7.

17 Rootman DS, Hasany SM, Basu PK. A morphometric study of endothelial cells of human corneas stored in MK media and warmed at $37^{\circ} \mathrm{C}$. Br J Ophthalmol 1988; 72: 545-9.

18 Stein RM, Bourne WM, Campbell RJ. Chondroitin sulfate for corneal preservation at $4^{\circ} \mathrm{C}$ : evaluation by electron microscopy. Arch Ophthalmol 1986; 104: 1358-61.

19 Bourne WM, Lindstrom RL, Doughman DJ. Endothelial cell survival on transplanted human corneas preserved by organ culture with $1.35 \%$ chondroitin sulfate. Am J Ophthalmol 1985; 100: 789-93.

20 Lindstrom RL, Doughman DJ, Skelnik DL, Mindrup EA. Minnesota system corneal preservation. Br J Ophthalmol 1986; 70: $47-54$.

21 Taylor $\mathrm{MJ}$. The role of $\mathrm{pH}$ and buffer capacity in the recovery of function of smooth muscle cooled to $-13^{\circ} \mathrm{C}$ in unfrozen media. Cryobiology 1982; 19: 585-601.

22 Taylor MJ, Pignat Y. Practical acid dissociation constants, temperature coefficients and buffer capacities for some biological buffers in solutions containing dimethyl sulfoxide between 25 and $-12^{\circ} \mathrm{C}$. Cryobiology 1982; 19: 99-109.

23 Gonnering R, Edelhauser HF, Van Horn DL, Durant W. pH tolerance of rabbit and human corneal endothelium. Invest Ophthalmol Vis Sci 1979: 18: 373-91.

24 Green K, Simon S, Kelly GM, Bowman KA. Effects of NA,$C l$, carbonic anhydrase, and intracellular $\mathrm{pH}$ on corneal endothelial bicarbonate transport. Invest Ophthalmol Vis Sci 1981; 21: 586-91.

25 Fischer FH, Wiederholt M. pH dependency of sodium and chloride transport in isolated human corneas. Invest Ophthalmol Vis Sci 1978; 17: 810-3.

26 Bowman KA, Elijah RD, Cheeks KE, Green K. Intracellular potential and $\mathrm{pH}$ of rabbit corneal endothelial cells. Curr Eye Res 1984: 3: 991-1000.

27 Fischer F, Voigt G, Liegl O, Wiederholt M. Effect of pH on potential difference and short circuit current in the isolated human cornea. Pflugers Arch 1974; 349: 119-31.

28 Hull DS, Green K. Bowman K, Csukas S, Riley MV. Intracellular $\mathrm{pH}$ and glutathione levels in rabbit corneal endothelium following storage in moist chamber and MK medium. Invest Ophthalmol Vis Sci 1983: 24: 214-7.

29 Tamaki K, Varnell ED, Kaufman HE. K-Sol corneal preservation at room temperature. Br J Ophthalmol 1988; 72: 370-6.

Accepted for publication 27 April 1989 